\title{
Bosworth-type fibular entrapment fracture of the ankle without dislocation: a rare case report and a review of the literature
}

\author{
Sang-Jin Han, Jong-Heon Kim, Du-Bin Yang, Boo-Seop Kim, Hyun-Soo Ok \\ Departments of Orthopedic Surgery, Hyundae General Hospital, Chung-Ang University, Namyangju-Si, Kyunggi-Do, Korea \\ Correspondence to: Hyun-Soo Ok, MD. Departments of Orthopedic Surgery, Hyundae General Hospital, Chung-Ang University, Namyangju-Si, \\ Kyunggi-Do, Korea. Email: mdohs@naver.com.
}

\begin{abstract}
Bosworth fracture-dislocation of ankle is a rare and irreducible type of ankle injury, with a high incidence of complication. This type of fracture was defined originally as entrapment of the proximal fragment of the fibula behind the posterior tubercle of the distal tibia. Recently, many variants of this type of fracture dislocation have been reported, but all of those reports included the syndesmosis ligament injury of ankle. Here, we report a case of a particularly rare variant of Bosworth fracture-dislocation without syndesmosis ligament injury of ankle. A 48-year-old male presented with a Bosworth fracture dislocation with entrapment of proximal fragment behind the tibia. After temporary treatment in emergency department was applied, emergency open reduction and internal fixation with a plate and screws was performed due to irreducibility of the fracture fragment. The fractured lateral malleolus was entrapped behind the tibia and rupture of the interosseous ligament was found intraoperatively. The anterior inferior tibiofibular ligament, a part of syndesmosis ligament of ankle, was grossly intact and no abnormal findings was seen by fluoroscopy with external rotational stress. Moreover, the deltoid ligament was found to be normal in ultrasonography. There were no complications after surgery and the patient showed full functional recovery at 2 years follow up. These fractures will frequently be irreducible and should be considered for open reduction and internal fixation with the careful evaluation of injury mechanisms with syndesmotic stability.
\end{abstract}

Keywords: Ankle fracture; Bosworth fracture; ankle dislocation; syndesmosis; case report

Submitted Jul 03, 2020. Accepted for publication Nov 26, 2020.

doi: $10.21037 /$ atm-20-5112

View this article at: http://dx.doi.org/10.21037/atm-20-5112

\section{Introduction}

Irreducible ankle fracture or fracture dislocation is a particularly rare condition $(1,2)$. There are many reasons that interfere in the reduction of fragments, including fracture dislocation of the tibiotalar joint as a result of anterior and posterior dislocation of the fibula, medial malleolar fracture with deltoid ligament or tibialis posterior tendon inter position, and tibiotalar diastasis with extensor tendon entrapment in the distal tibiofibular joint, restricted by the extensor retinaculum (1-3). Among these, Bosworth (4) described 5 cases of an unusual fracture-dislocation of the ankle with entrapment of the proximal fragment of the fibula behind the posterior tubercle of the distal tibia. Most of these lesions were induced by supination and external rotation injury patterns according to the LaugeHansen classification and it was well known to be missed diagnosis easily (5-7). This type of ankle fracture is usually unable to be reduced in a closed fashion and should be treated with surgical reduction to prevent articular and soft tissue damage as a result of repeated attempts of closed reduction (6-8). In previous studies, many cases have been reported of this unusual type of fracture, but most cases are accompanied by ankle dislocation and syndesmosis injuries which needs combined treatment $(1,2,5,8-10)$. To the best of our knowledge, there is yet to be a report of a lateral malleolar fracture with locked the proximal fragment behind the distal tibia without ankle dislocation or syndesmosis injury. Recently, we experienced an unusual variant case of a Bosworth type fracture without dislocation or syndesmosis 

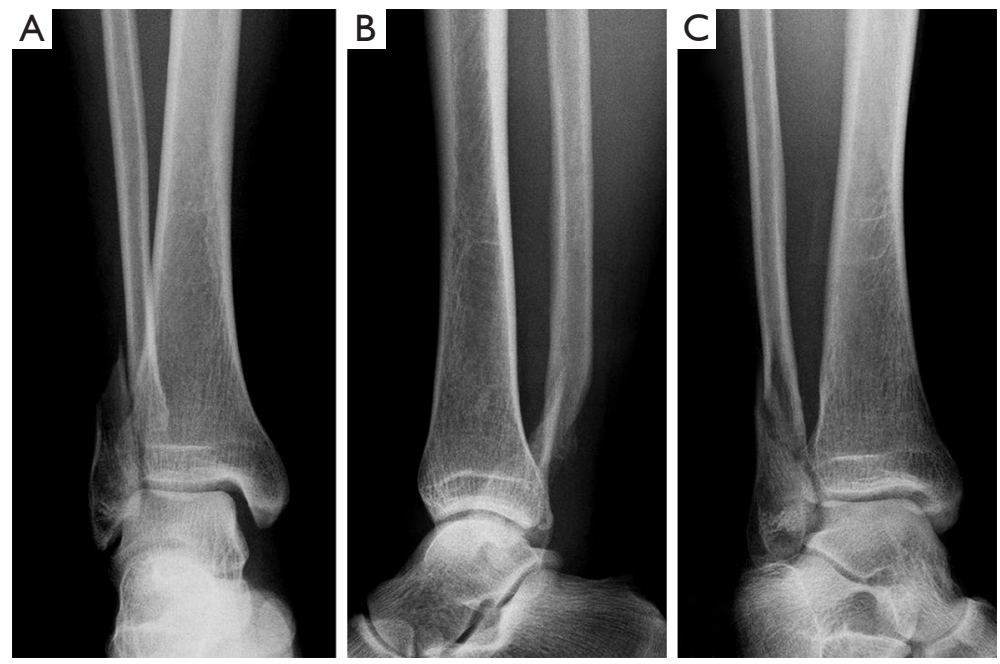

Figure 1 Preoperative radiographs of the anteroposterior, lateral, and mortice ankle. (A) The fracture of the distal fibula was classified as Danis-Weber type B, typical overlap of the distal tibia and fibula in anteroposterior view. (B) Intact joint alignment was shown in lateral view. (C) Medial clear space is equal to the superior clear space between the talus and the distal tibia in mortice view.
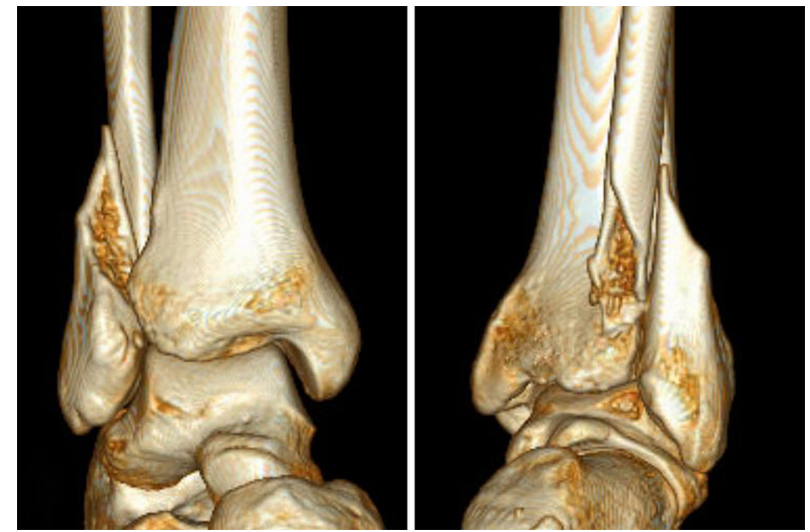

Figure 2 3D reconstruction of computed tomography (CT) scan shows posterior dislocation of the proximal fragment of the fibula and its entrapment behind the posterior tubercle of the distal tibia.

injury. Therefore, we herein present the following case in accordance with the CARE reporting checklist along with a literature review of the topic (available at http://dx.doi. org/10.21037/atm-20-5112).

\section{Case presentation}

All procedures performed in studies involving human participants were in accordance with the ethical standards of the institutional and/or national research committee(s) and with the Helsinki Declaration (as revised in 2013).
Written informed consent was obtained from the patient for publication of this study and any accompanying images. A 48-year-old male presented with an injured ankle after slipping over when coming down a mountain. Examination revealed slight ankle swelling but no deformity, bruising on the medial deltoid ligament area, and no motion was possible due to pain. Tender points were identified on both sides of the ankle and pain was equivocal on the anterior inferior tibiofibular ligament area. Peripheral pulses and nerve innervations were intact. The anteroposterior view showed an overlap of the distal fibula and tibia, a fracture in the level of syndesmosis (Danis-Weber type B). The lateral view showed intact joint alignment of the ankle and medial and superior clear space of the tibiotalar joint were equal in the mortice view (Figure 1). These findings were confirmed by a 3 -dimentianal (3D) reconstruction computed tomography (CT) scan of the ankle (Figure 2). As many cases of Bosworth type fractures are irreducible or not anatomic, a closed reduction was not attempted.

After temporary treatment was applied in the emergency department, an emergency open reduction and internal fixation $(\mathrm{OR} / \mathrm{IF})$ was carried out in operation room. During surgery, the locked fibula was reduced using a bone holder while retracting the ankle. A fractured lateral malleolus entrapped behind the tibia and a rupture of the interosseous ligament was revealed intraoperatively, but the anterior inferior tibiofibular ligament was intact (Figures 3 and 4). Internal fixation of the fibular fracture was accomplished by 


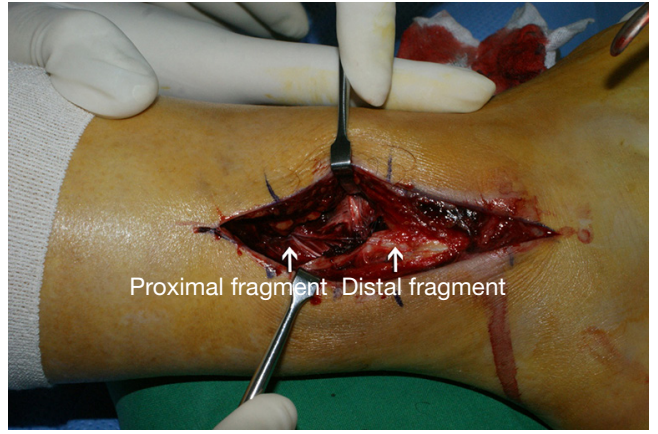

Figure 3 The proximal fragment of the lateral malleolus entrapped behind the tibia and rupture of the interosseous ligament were seen intraoperatively.

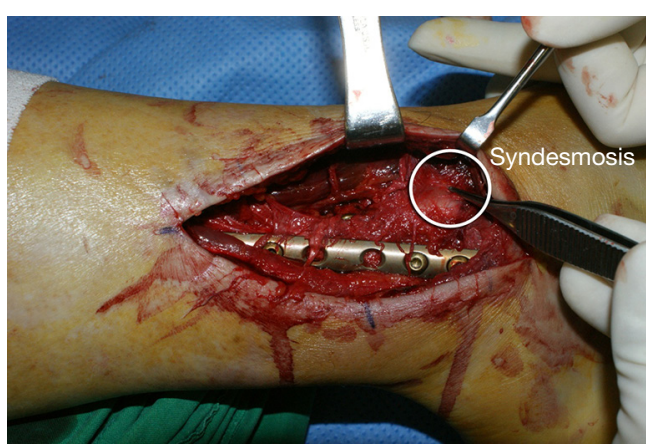

Figure 4 After reduction and internal fixation, the anterior inferior tibiofibular ligament was checked and revealed to be intact.

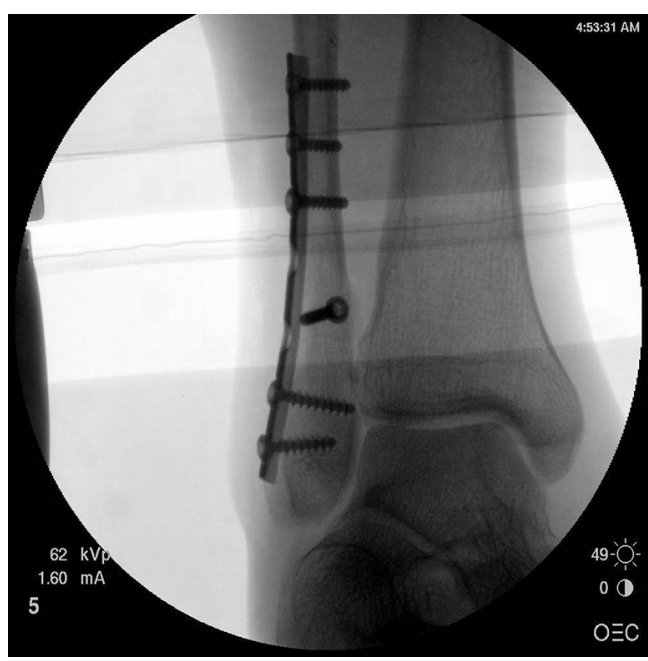

Figure 5 Intraoperative fluoroscopy images of the anteroposterior view with external rotation stress to the ankle. In plantar flexionexternal rotation position.

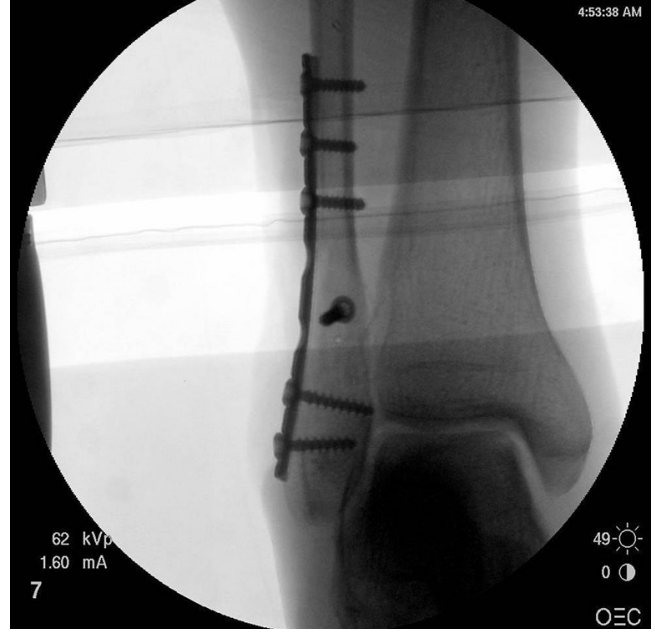

Figure 6 In dorsiflexion position-external rotation position, there was no widening of clear space at the distal tibiofibular joint.

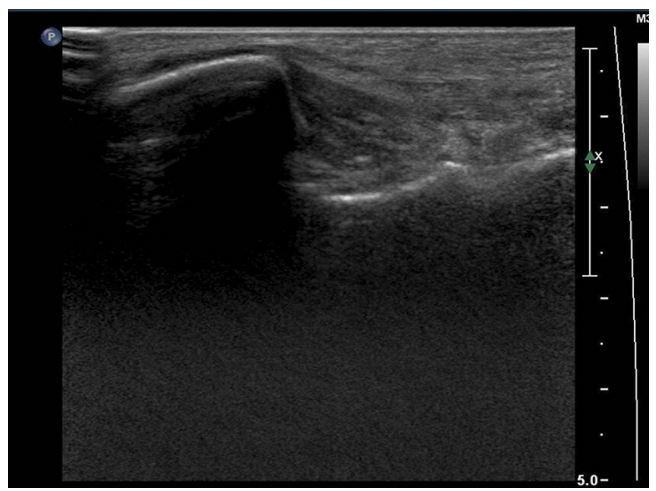

Figure 7 Ultrasonography showed an intact deltoid ligament.

interfragmentary lag screw and a one-third tubular plate. After fixation, intraoperative fluoroscopy of the planta- and dorsiflexion position with external rotation stress was used to evaluate syndesmotic stability, resulting in no widening of the clear space (Figures 5 and 6 ). As the ankle mortice was in anatomic position, as revealed by intraoperative imaging, the deltoid ligament was not approached. Seven days after the surgery, ultrasonography showed the intact deltoid ligament despite bruising on the medial side of the ankle (Figure 7). Postoperatively, a short-leg cast was worn for 6 weeks, and the fracture was completely healed at 2-year follow up (Figure 8). The patient showed full functional recovery of the ankle and no subjective symptoms. 

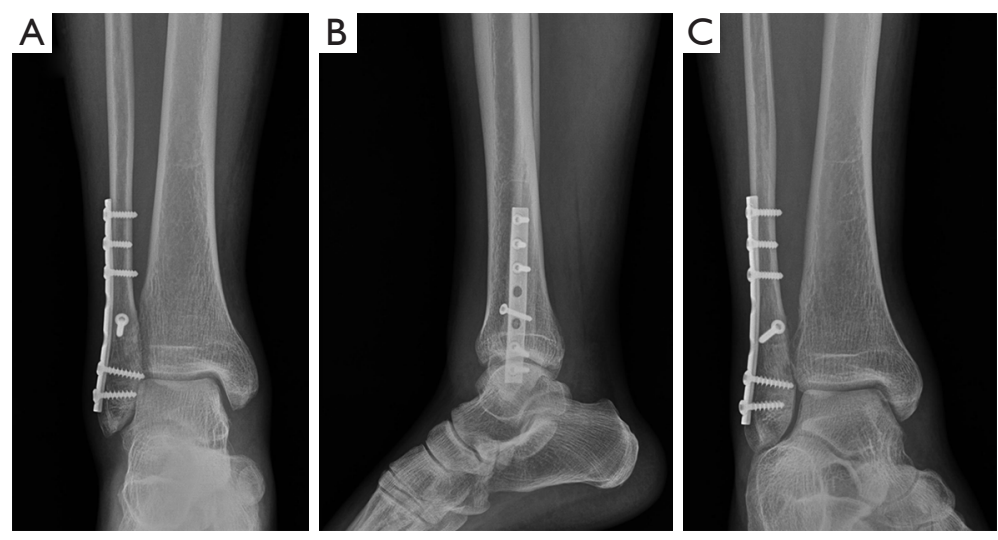

Figure 8 Postoperative follow up radiographs. (A) Anteroposterior view; (B) lateral view; (C) mortice view.

\section{Discussion}

This case of an unusual variant of a rare type of ankle fracture-dislocation accounts for around $1.6 \%$ of ankle fractures (8). A Bosworth fracture-dislocation has been defined as a fixed posterior fracture-dislocation of the distal part of the fibula in which the proximal fibular shaft fragment locks behind the posterior tibial tubercle, which is very rare phenomenon $(1,2,8)$. This fracture and the associated tibiotalar dislocations are typically irreducible by closed means, and compartment syndrome or skin necrosis could occur if the reduction procedure was delayed. Moreover, persistent pain and functional deficit would be followed if the syndesmosis injury was left untreated $(1,10,11)$.

The mechanism of injury of fracture-dislocation was first described by Bosworth and later by Perry et al. (12) As a generally accepted mechanism, an external rotation force applied to a supinated foot after dividing the anterior and posterior tibiofibular ligament produces the entrapped fibula behind the posterolateral ridge of the tibia (12).

The morphology of Bosworth fracture lesions varies and many patients sustain a fracture of the fibula at the level of syndesmosis (4). Supra-syndesmotic fractures of the fibula with posterior entrapment of the fibula have been described by Mayer and Evarts (3) and Molinari and colleagues (13). In the case report by Williams et al. (9), a case of Bosworth dislocation without ankle fracture was described, while Ren et al. (5) also reported rare variants of Bosworth fracturedislocation with adduction medial malleolar fractures, of which the injury mechanisms are different from that of the original Bosworth ankle fracture-dislocation. Although Bosworth type fracture-dislocation have been revealed to be a severe ankle injury including various fractures and ligament structures $(1,2,5,10)$, early interventions of these lesions show good clinical outcomes $(8,14)$. In recent study, the prevalence of Bosworth fracture-dislocations was found as $1.62 \%(51 / 3,140$ cases), which seems to be higher than author expected, and the postoperative outcomes were significantly better in early intervention patients (8).

To the best of our knowledge, this is the first report to date which involves a fibula fracture at the level of syndesmosis with posterior entrapment behind the posterior tubercle of the tibia with stable syndesmosis and deltoid ligament. As a general belief of the Bosworth mechanism, syndesmosis rupture should be found first to dislocate the fibula which results in syndesmosis instability. However, intraoperative fluoroscopic evaluation of this patient revealed that the distal tibiofibular joint was stable, considered similar to the supination-external rotation stage II in the Lauge-Hansen classification system (15) with stable syndesmosis (Figures 5 and 6 ). While relevant syndesmotic injuries are less frequent in supination-external rotation ankle fractures, at the rate of $17-30 \%(14,16,17)$, this patient may not have injured the syndesmosis even though suffering from a Bosworth type fracture.

In the cadaveric study, pronation-external rotation followed by dorsiflexion induced this type of fracture. Since dorsiflexion of the ankle with axial loading also causes lateral displacement of ankle $(11,18)$, sequential loading of force could lead to posterior dislocation and entrapment of the fibula. First, pronation-external rotation injury occurred by being trampled by a rock, which caused a fibular fracture not unlike supination-external rotation type II in the LaugeHansen classification system (15). Afterward, axial force with dorsiflexion of the ankle followed which produced 


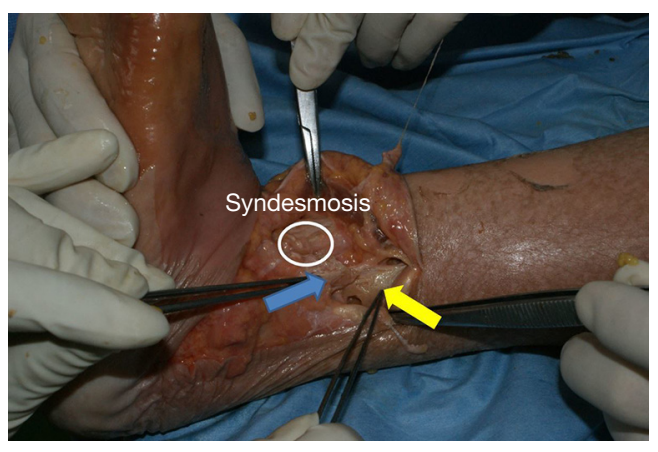

Figure 9 In cadaveric examination, pronation external rotation followed by dorsiflexion could induce Bosworth type fracture with stable syndesmosis in left ankle (white circle: syndesmosis ligament, blue arrow: distal fragment of fibula, yellow arrow: proximal fragment of fibula).

more lateral displacement of distal fibula, which led to the proximal fragment of fibula migrating to the relatively medial side, resulting in this being located behind the tibial tuberosity (Figure 9).

In the study by Haraguchi et al. (19), pronation-external rotation injury could cause the same type of supinationexternal rotation fracture in the Lauge-Hansen classification system, we suggest that the injury mechanism of this case could be explained as an external-rotation injury followed by dorsiflexion, regardless of supination or pronation of foot. Although Bosworth fractures occur as high energy trauma, this case occurred as a combination of low energy trauma.

Since the standard methods to evaluate syndesmosis stability are not defined, distal tibiofibular joint stability was assessed by intraoperative fluoroscopy only. For accurate evaluation, magnetic resonance imaging (MRI) would be more useful than fluoroscopy $(20,21)$. Finally, further biomechanical study would be required to evaluate the injury mechanism, not only simultaneous events, but also sequential injuries with a combination of different forces, even low energy trauma.

In brief, there might be a possibility of Bosworth type fracture-dislocation without syndesmosis injury, the treatment to the syndesmosis should be considered after careful evaluation of distal tibiofibular joint stability to avoid unnecessary surgery.

\section{Conclusions}

In conclusion, the variant of Bosworth fracture-dislocation presented here is a rare but a severe injury of the ankle and the unusual presentation of the fracture could be aroused with the sequential application of injury forces. These fractures will frequently be irreducible and should be considered for open reduction and internal fixation with the careful evaluation of injury mechanisms with syndesmotic stability.

\section{Acknowledgments}

Funding: None.

\section{Footnote}

Reporting Checklist: The authors have completed the CARE reporting checklist. Available at http://dx.doi.org/10.21037/ atm-20-5112

Conflicts of Interest: All authors have completed the ICMJE uniform disclosure form (available at http://dx.doi. org/10.21037/atm-20-5112). The authors have no conflicts of interest to declare.

Ethical Statement: The authors are accountable for all aspects of the work in ensuring that questions related to the accuracy or integrity of any part of the work are appropriately investigated and resolved. All procedures performed in studies involving human participants were in accordance with the ethical standards of the institutional and/or national research committee(s) and with the Helsinki Declaration (as revised in 2013). Written informed consent was obtained from the patient for publication of this manuscript and any accompanying images.

Open Access Statement: This is an Open Access article distributed in accordance with the Creative Commons Attribution-NonCommercial-NoDerivs 4.0 International License (CC BY-NC-ND 4.0), which permits the noncommercial replication and distribution of the article with the strict proviso that no changes or edits are made and the original work is properly cited (including links to both the formal publication through the relevant DOI and the license). See: https://creativecommons.org/licenses/by-nc-nd/4.0/.

\section{References}

1. Bartonícek J, Fric V, Svatos F, et al. Bosworth-type fibular entrapment injuries of the ankle: the Bosworth lesion. A 
report of 6 cases and literature review. J Orthop Trauma 2007;21:710-7.

2. Foldager CB, Barckman J, Robertsen K, et al. Bosworth fracture dislocation of the ankle: - Two case reports with perioperative illustration. Trauma Case Rep 2018;17:39-42.

3. Mayer PJ, Evarts CM. Fracture-dislocation of the ankle with posterior entrapment of the fibula behind the tibia. J Bone Joint Surg Am 1978;60:320-4.

4. Bosworth DM. Fracture-dislocation of the ankle with fixed displacement of the fibula behind the tibia. J Bone Joint Surg Am 1947;29:130-5.

5. Ren W, Hu YC, Lu JK. Rare variants of Bosworth fracture-dislocation: Bosworth fracture-dislocation with medial malleolus adduction type fracture. Chin J Traumatol 2019;22:120-4.

6. Cappuccio M, Leonetti D, Di Matteo B, et al. An uncommon case of irreducible ankle fracture-dislocation: the "Bosworth-like" tibio-fibular fracture. Foot Ankle Surg 2017;23:e1-e4.

7. Yang KH, Won Y, Lim JR, et al. Assessment of Bosworthtype fracture by external oblique radiographs. Am J Emerg Med 2014;32:1387-90.

8. Won Y, Lee GS, Hwang JM, et al. Improved functional outcome after early reduction in Bosworth fracturedislocation. Foot Ankle Surg 2019;25:798-803.

9. Williams AD, Blue M, Douthit C, et al. Bosworth Dislocation without Associated Fracture. Case Rep Orthop 2018;2018:7284643.

10. Kostlivý K, Bartoníček J, Rammelt S. Posterior malleolus fractures in Bosworth fracture-dislocations. A combination not to be missed. Injury 2020;51:537-41.

11. Beumer A, Valstar ER, Garling EH, et al. Effects of ligament sectioning on the kinematics of the distal tibiofibular syndesmosis: a radiostereometric study of

Cite this article as: Han SJ, Kim JH, Yang DB, Kim BS, Ok HS. Bosworth-type fibular entrapment fracture of the ankle without dislocation: a rare case report and a review of the literature. Ann Transl Med 2021;9(2):178. doi: 10.21037/atm$20-5112$
10 cadaveric specimens based on presumed trauma mechanisms with suggestions for treatment. Acta Orthop 2006;77:531-40.

12. Perry CR, Rice S, Rao A, Burdge R. Posterior fracturedislocation of the distal part of the fibula. Mechanism and staging of injury. J Bone Joint Surg Am 1983;65:1149-57.

13. Molinari M, Bertoldi L, De March L. Fracture dislocation of the ankle with the fibula trapped behind the tibia. A case report. Acta Orthop Scand 1990;61:471-2.

14. Kortekangas TH, Pakarinen HJ, Savola O, et al. Syndesmotic fixation in supination-external rotation ankle fractures: a prospective randomized study. Foot Ankle Int 2014;35:988-95.

15. Lauge-Hansen N. Fractures of the ankle. II. Combined experimental-surgical and experimental-roentgenologic investigations. Arch Surg 1950;60:957-85.

16. Jenkinson RJ, Sanders DW, Macleod MD, et al. Intraoperative diagnosis of syndesmosis injuries in external rotation ankle fractures. J Orthop Trauma 2005;19:604-9.

17. Weening B, Bhandari M. Predictors of functional outcome following transsyndesmotic screw fixation of ankle fractures. J Orthop Trauma 2005;19:102-8.

18. Kellett JJ. The clinical features of ankle syndesmosis injuries: a general review. Clin J Sport Med 2011;21:524-9.

19. Haraguchi N, Armiger RS. A new interpretation of the mechanism of ankle fracture. J Bone Joint Surg Am 2009;91:821-9.

20. Hermans JJ, Beumer A, Hop WC, et al. Tibiofibular syndesmosis in acute ankle fractures: additional value of an oblique MR image plane. Skeletal Radiol 2012;41:193-202.

21. Hermans JJ, Wentink N, Beumer A, et al. Correlation between radiological assessment of acute ankle fractures and syndesmotic injury on MRI. Skeletal Radiol 2012;41:787-801. 